\title{
The challenge of MOEMS deformable mirror tested in cryo for future AO systems
}

\author{
Frederic Zamkotsian ${ }^{1}$, Patrick Lanzoni ${ }^{1}$, Rudy Barette ${ }^{1}$, \\ Michael Helmbrecht ${ }^{2}$, Franck Marchis ${ }^{2}$, Alex Teichman ${ }^{2}$ \\ ${ }^{1}$ Aix Marseille Univ, CNRS, LAM, Laboratoire d'Astrophysique de Marseille, \\ 38 rue Frederic Joliot Curie, 13388 Marseille Cedex 13, France \\ ${ }^{2}$ Iris AO, 2930 Shattuck Avenue, Berkeley, CA 94705, USA \\ e-mail: frederic.zamkotsian@lam.fr
}

\begin{abstract}
MOEMS Deformable Mirrors (DM) are key components for next generation optical instruments implementing innovative adaptive optics systems, in existing telescopes and in the future ELTs. Characterizing these components well is critical for next generation instruments. This is done by interferometry, including surface quality measurement in static and dynamical modes, at ambient and in vacuum/cryo. We use a compact cryo-vacuum chamber designed for reaching 10-6 mbar and $160 \mathrm{~K}$, in front of our custom Michelson interferometer, able to measure performance of the DM at actuator/segment level and at the entire mirror level, with a lateral resolution of $2 \mu \mathrm{m}$ and a sub-nanometer z-resolution. We tested the PTT 111 DM from Iris AO: an array of single crystalline silicon hexagonal mirrors with a pitch of $606 \mu \mathrm{m}$, able to move in tip, tilt and piston (stroke $5-7 \mu \mathrm{m}$, tilt $+/-5 \mathrm{mrad}$ ). The device could be operated successfully from ambient to $160 \mathrm{~K}$. An additional, mainly focus-like, $500 \mathrm{~nm}$ deformation of the entire mirror is measured at $160 \mathrm{~K}$; we were able to recover the best flat in cryo by correcting the focus and local tip-tilts on all segments, reaching $12 \mathrm{~nm}$ rms. Finally, the goal of these studies is to test DMs in cryo and vacuum conditions as well as to improve their architecture for stable operation in harsh environment.
\end{abstract}

Keywords: micromirror array, MOEMS, cryogenic testing, adaptive optics, wavefront correction.

\section{INTRODUCTION}

Several research groups around the world are currently involved in the design of highly performing adaptive optical (AO) systems as well as for next generation instrumentation of $10 \mathrm{~m}$-class telescopes than for future extremely large optical telescopes.

Wavefront correction like adaptive optics systems are based on a combination of three elements, the wavefront sensor for the measurement of the shape of the wavefront arriving in the telescope, the deformable mirror is the correcting element, and finally the real time computer closing the loop of the system at a frequency ranging from 0.5 to $3 \mathrm{kHz}$, in order to follow the evolution of the atmospherical perturbations (Fig. 1).

Four main types of AO systems have been built or are under development: Single-Conjugate Adaptive Optics (SCAO), Multi-Conjugate Adaptive Optics (MCAO), Multi-Object Adaptive Optics (MOAO), and Extreme Adaptive Optics (ExAO). These AO systems are associated with different types of WaveFront Sensors (WFS), combined with natural guide stars or laser guide stars, and different architectures of Deformable Mirrors (DM). Numerous science cases will use these AO systems, SCAO, the "classical" AO system will provide accurate narrow field imagery and spectroscopy, $\mathrm{MCAO}$, wide field imagery and spectroscopy, MOAO, distributed partial correction AO, and high dynamic range AO for the detection and the study of circumstellar disks and extra-solar planets. Corrected fields will vary from few arcsec to several arcmin.

These systems require a large variety of deformable mirrors with very challenging parameters. For a $8 \mathrm{~m}$ telescope, the number of actuators varies from a few 10 up to 5000; these numbers increase impressively for a $40 \mathrm{~m}$ telescope, ranging 
from a few 100 to over 50000 , the inter-actuator spacing from less than $200 \mu \mathrm{m}$ to $1 \mathrm{~mm}$, and the deformable mirror size from $10 \mathrm{~mm}$ to a few $100 \mathrm{~mm}$. Conventional technology cannot provide this wide range of deformable mirrors. The development of new technologies based on micro-opto-electro-mechanical systems (MOEMS) is promising for future deformable mirrors. The major advantages of the micro-deformable mirrors (MDM) are their compactness, scalability, and specific task customization using elementary building blocks. This technology permits the development of a complete generation of new mirrors. However this technology has also some limitation. For example, pupil diameter is an overall parameter and for a $40 \mathrm{~m}$ primary telescope, the internal pupil diameter cannot be reduced below $0.5 \mathrm{~m}$. According to the maximal size of the wafers ( 8 inches), a deformable mirror based on MOEMS technology cannot be build into one piece. New AO architectures have been proposed to avoid this limitation. ${ }^{1}$

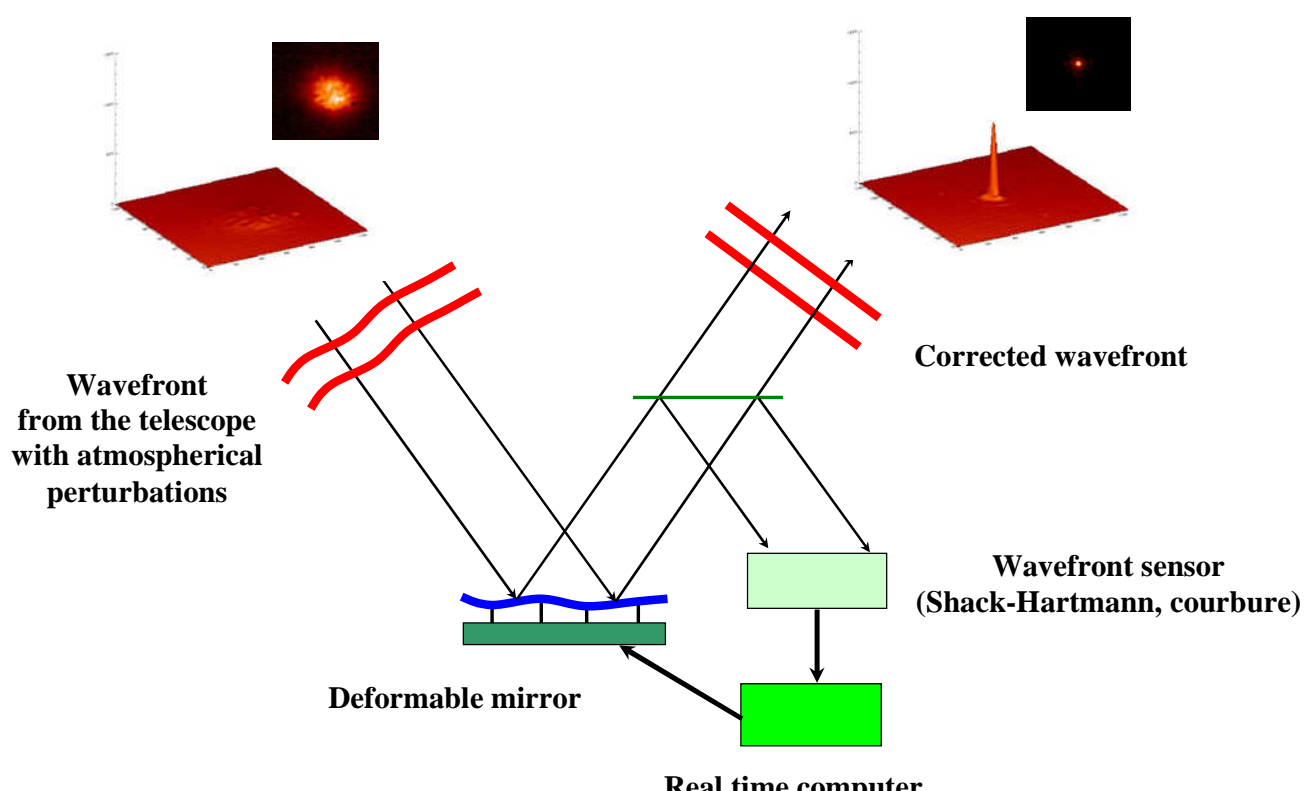

Fig. 1: Schematic of a wavefront correction system

LAM is involved since several years in conception of new MOEMS devices as well as in characterization of these components for the future instrumentation of ground-based and space telescopes. These studies include programmable slits for application in multi-object spectroscopy (JWST, European networks, EUCLID, BATMAN), deformable mirrors for adaptive optics, and programmable gratings for spectral tailoring.

We are particularly engaged in a European development of micromirror arrays (MMA) called MIRA for generating reflective slit masks in future Multi-object spectroscopy (MOS) instruments; this technique is a powerful tool for space and ground-based telescopes for the study of the formation and evolution of galaxies. MMA with $100 \times 200 \mu \mathrm{m}^{2}$ singlecrystal silicon micromirrors were successfully designed, fabricated and tested. Arrays are composed of 2048 micromirrors $(32 \times 64)$ with a peak-to-valley deformation less than $10 \mathrm{~nm}$, a tilt angle of $24^{\circ}$ for an actuation voltage of $130 \mathrm{~V}$. The micromirrors were actuated successfully before, during and after cryogenic cooling, down to $162 \mathrm{~K}$. The micromirror surface deformation was measured at cryo and is below $30 \mathrm{~nm}$ peak-to-valley. ${ }^{2,3}$ In order to fill large focal planes (mosaicing of several chips), we are currently developing large micromirror arrays integrated with their electronics.

LAM is also leading the conception and realization of new MOEMS-based instruments. We are developing a 2048x1080 Digital-Micromirror-Device-based (DMD) MOS instrument to be mounted on the Telescopio Nazionale Galileo (TNG) and called BATMAN. A two-arm instrument has been designed for providing in parallel imaging and spectroscopic capabilities. BATMAN on sky is of prime importance for characterizing the actual performance of this new family of MOS instruments, as well as investigating new observational modes on astronomical objects, from faint and remote galaxies to active areas in nearby galaxies and small bodies of the solar system. This instrument will be placed at TNG by end-2018. ${ }^{4}$ 
In this paper, we present the interferometric characterization of a segmented deformable mirror from Iris AO. The results on the mirror surface measured at ambient down to $160 \mathrm{~K}$ are shown and analysed: the mirror best flat condition is obtained at ambient and at $160 \mathrm{~K}$.

\section{DEFORMABLE MIRRORS}

Three main Micro-Deformable Mirrors (MDM) architectures are under study in different laboratories and companies. First, the bulk micro-machined continuous-membrane deformable mirror, studied by Delft University and OKO company, is a combination of bulk silicon micromachining with standard electronics technology $y^{5}$. This mirror is formed by a thin flexible conducting membrane, coated with a reflective material, and stretched over an electrostatic electrode structure. This mirror shows a very good mirror quality, but the mean deformed surface is a concave surface, and the number of actuators cannot be scalable to hundreds of electrodes. Second, the segmented, micro-electro-mechanical deformable mirror realized by Iris $\mathrm{AO}^{6}$ consists of a set of segmented piston-tip-tilt moving surfaces, fabricated in dense array. For adaptive optics application, the wavefront has to be properly sampled, increasing the number of actuators for a given number of modes to be corrected. Third, the surface micro-machined continuous-membrane deformable mirror made by Boston Micromachines Corporation (BMC) is based on a single compliant optical membrane supported by multiple attachments to an underlying array of surface-normal electrostatic actuators ${ }^{7}$. The efficiency of this device has been demonstrated recently in several AO system, including the GPI instrument on Gemini telescope. The third concept is certainly the most promising architecture, but it shows limited strokes for large driving voltages, and mirror surface quality may need further improvement for Extreme AO. All these devices are based on silicon or polysilicon materials.

\subsection{BMC Deformable Mirror}

BMC produces the most advanced MEMS deformable mirrors. The concept is based on an array of electrostatic actuators linked one by one to a continuous top mirror (Fig. 2). Their main parameters are approaching the requirements values, i.e. large number of actuators (up to 4096, see Fig. 2), large stroke (up to $5.5 \mu \mathrm{m}$ ), good surface quality, but they still need large voltages for their actuation $(150-250 \mathrm{~V})$.

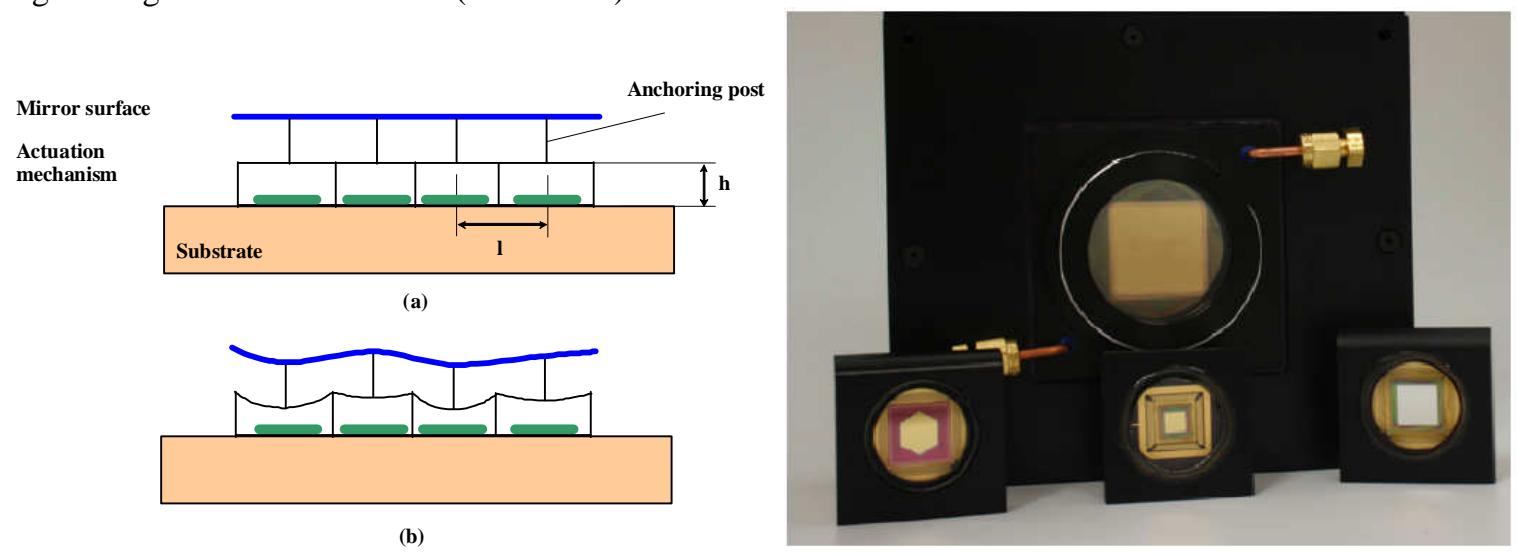

Fig. 2: Continuous membrane MDM from BMC

\subsection{Iris AO Deformable Mirror}

IRIS AO is producing segmented piston tip tilt mirrors with very flat mirrors. An exploded-view schematic diagram is presented in Fig. 3a. The DM array is paved by 37 hexagonal segments with a size of $700 \mu \mathrm{m}$ from vertex-to-vertex, with a $606 \mu \mathrm{m}$ pitch. The segment is capable of moving in piston/tip/tilt motions (PTT). In Fig. 3a, scaling is highly exaggerated in the vertical direction. In Fig. $3 \mathrm{~b}$ is shown a die photograph of a 111-actuator 37-piston/tip/tilt-segment DM with $3.5 \mathrm{~mm}$ inscribed aperture (PTT111 device). The DM is manufactured using typical MEMS and integrated circuit materials such as polycrystalline silicon (polysilicon), silicon dioxides, silicon nitrides, and a proprietary bimorph material with similar coefficient of thermal expansion (CTE) to that of polysilicon. The s-shape of the bimorph flexures that elevates the DM segment is a result of engineered residual tensile stresses in the bimorph and actuator-platform polysilicon. After the DM is fabricated using highly stable MEMS materials, it is mounted onto a ceramic pin-grid array (PGA) package using an epoxy. The DM is sealed in nitrogen by epoxying a cover window over the DM. 

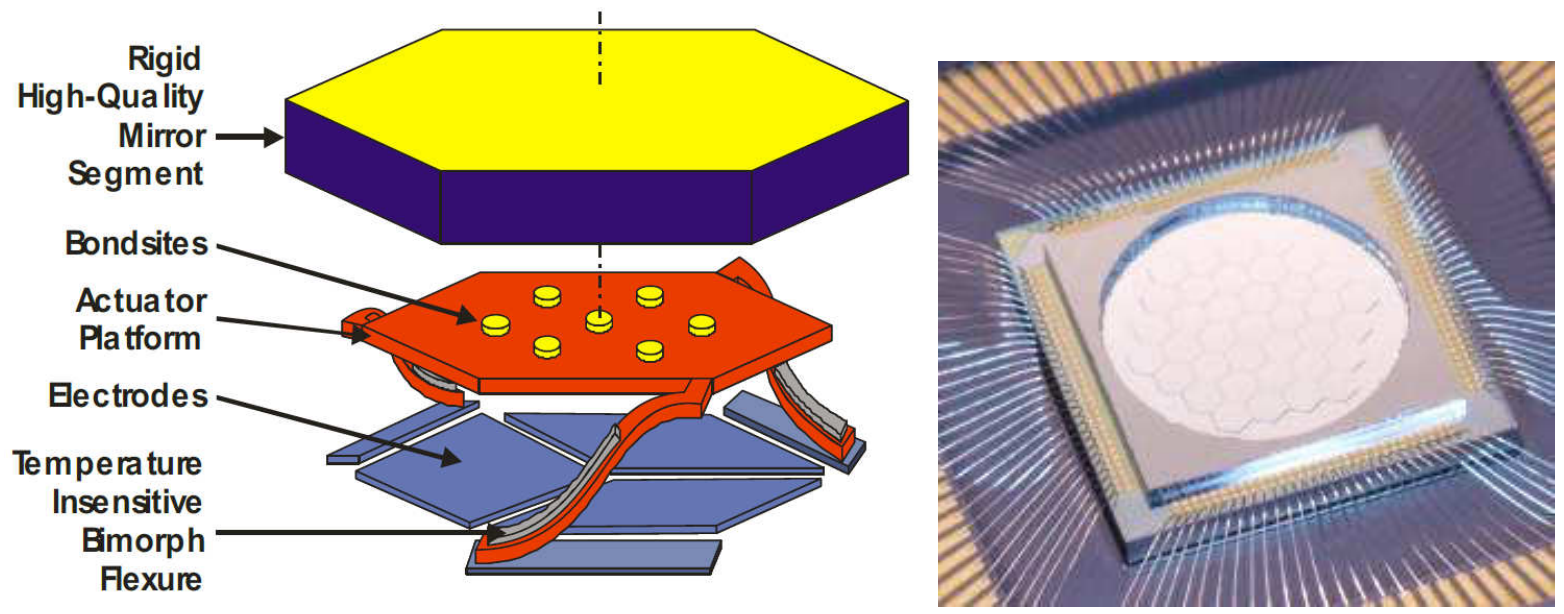

Fig. 3: Segmented MDM from Iris AO (a) Concept of one segment of the mirror;

(b) Die photograph of a 111-actuator 37-piston/tip/tilt-segment DM with $3.5 \mathrm{~mm}$ inscribed aperture (PTT111 device)

To actuate the DM, the red actuator-platform layer is held at ground potential and the three diamond-shaped electrodes are energized at different electrical potentials. Applying the same voltage to all three electrodes pulls the segment in a piston motion toward the electrodes. A differential voltage across the electrodes results in tip and tilt motions. Because the positioning is highly repeatable, the DM segment motion can be calibrated, thus linearizing the DM position into orthogonal coordinates.

\section{CRYOGENIC INTERFEROMETRIC TEST SET-UP}

The Laboratoire d'Astrophysique de Marseille has developed over the last few years an expertise in the characterization of micro-optical components. Our expertise in small-scale deformation characterization on the surface of micro-optical components has been conducted initially within the framework of the NASA study of a multi-object infrared spectrograph equipped with MOEMS-based slit masks for the JWST.

An interferometric characterization bench has been developed in order to measure the shape and the deformation parameters of these devices. All optical characterizations in static or dynamic behavior are performed, including measurements of optical surface quality at different scales, actuators stroke, maximum mirror deformation and cut-off frequency. This bench is a high-resolution and low-coherence Twyman-Green interferometer; out-of-plane measurements are performed with phase-shifting interferometry showing very high resolution (standard deviation<1nm). ${ }^{8}$

Cryogenic characterization was carried out in a custom built cryogenic chamber installed in front of our interferometric setup. The cryo-chamber has a pressure as low as $10 \mathrm{e}-6$ mbar and is able to cool down to $100 \mathrm{~K}$, when not loaded, using a cryogenic generator. ${ }^{9}$

The PTT111 device is packaged in PGA chip carrier and mounted in the cryo test set-up. The chamber is then closed by a flange and placed in front of the interferometer. Along the reference path, two compensation plates are placed for compensating the chamber window and the device window. By this way, we keep a high contrast for the interferometric fringes. ${ }^{10}$

\section{PTT111 SURFACE CHARACTERIZATION}

Our experiment is done on an engineering grade device where the segments \#23 and \#24 are lockouts. The segment thickness is $25 \mu \mathrm{m}$ and the coating is protected silver. The maximum array stroke is $3.01 \mu \mathrm{m}$, and the maximum tilt angle 
is 5 mrad. Fig. 4 is a picture of the device made on our bench (without the interferometric fringes). The two lockouts segments are at the upper right.

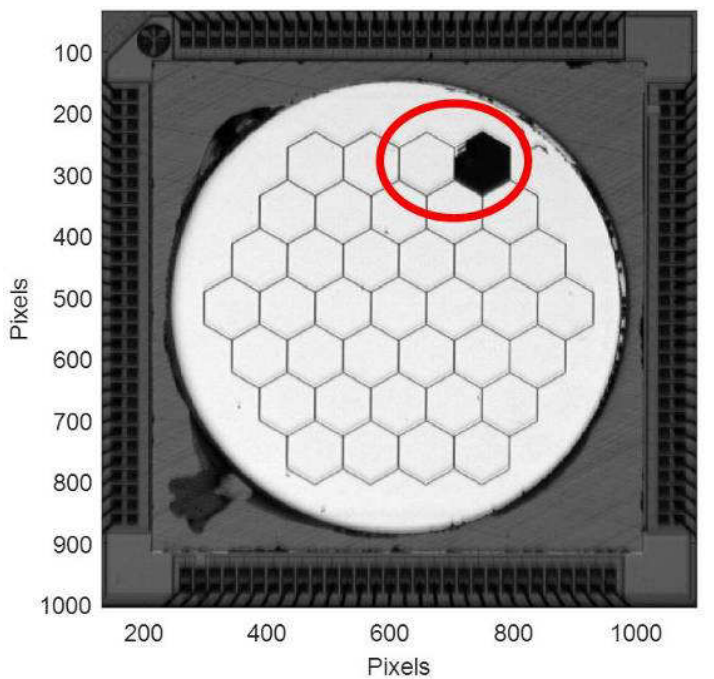

Fig. 4: PTT111 device tested in our experiment (engineering grade device) Segments \#23 and \#24 (in the red circle) are lockouts.

The device is driven either by the Graphical User Interface (GUI) provided by Iris-AO for the integration and precharacterization phases. The interferometric measurements are done with the LAM-developed software, in Matlab, and linked with the Matlab driver provided by Iris AO. The GUI is showing a view of the mirror with numbered segments, and global Zernike coefficient as well as local (at segment level) Zernike coefficients could be tuned for each actuator/segment.

A calibration step has been done by Iris AO for measuring each actuator response ( 3 actuators /segment). Then, a "best flat" condition is calculated in order to minimize the residual wavefront error on the surface, and applied. In Fig. 5 is a screen shot made at the beginning of the experiment when the best flat condition is applied; at left hand side, the interferometric image of the mirror with "best flat" condition; at right bottom, view of the Iris AO GUI for driving the device.

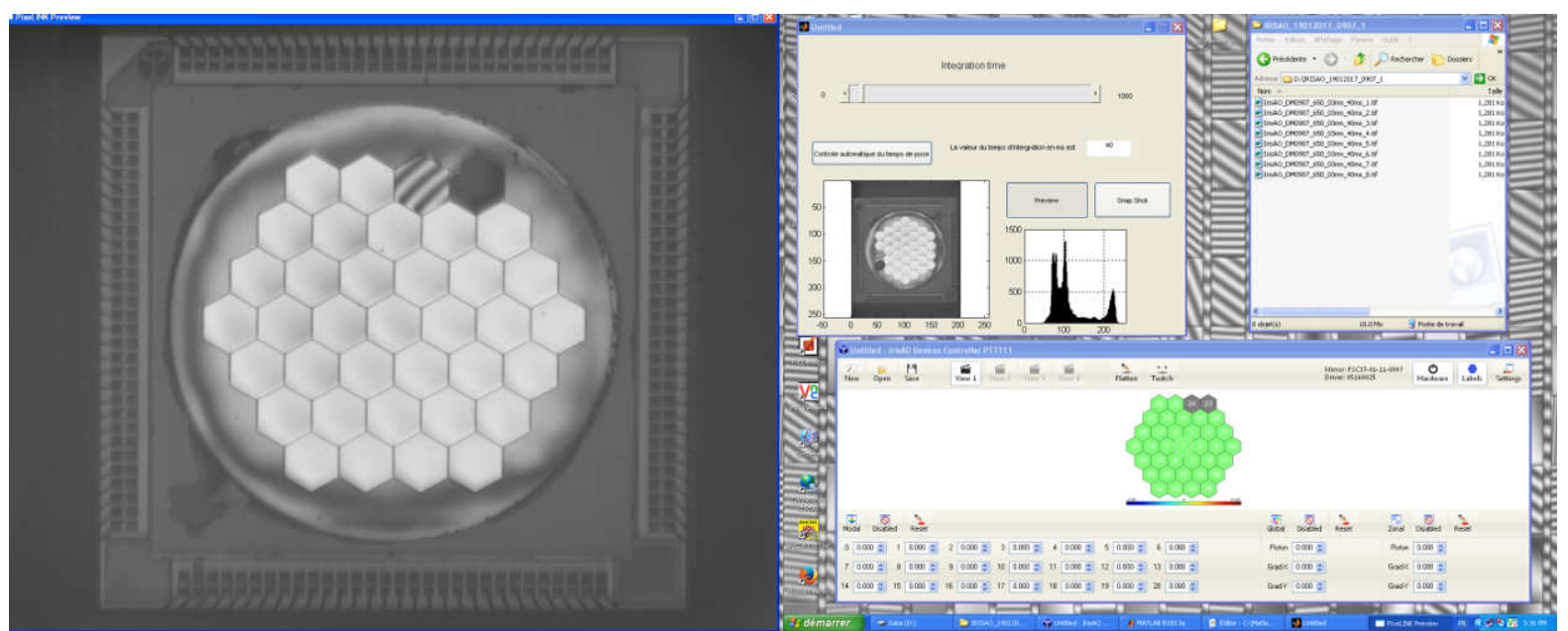

Fig. 5: Screen shot during the experiment; left: interferometric image of the mirror when the "best flat" condition is applied; right bottom: Iris AO GUI for driving the device.

We could then apply a serial of commands on the mirror. In Fig. 6, different mirror configurations are presented. From top left to bottom right, we can see:

- $\quad$ a pure piston $(150 \mathrm{~nm})$ on the central M1 segment, 
- a global astigmatism on the mirror, using the global Zernike coefficient set at 0.125

- a serial of three identical tilts on all segments in X direction, with $0.25 \mathrm{mrad}, 1.5 \mathrm{mrad}$ and $4.9 \mathrm{mrad}$ respectively.
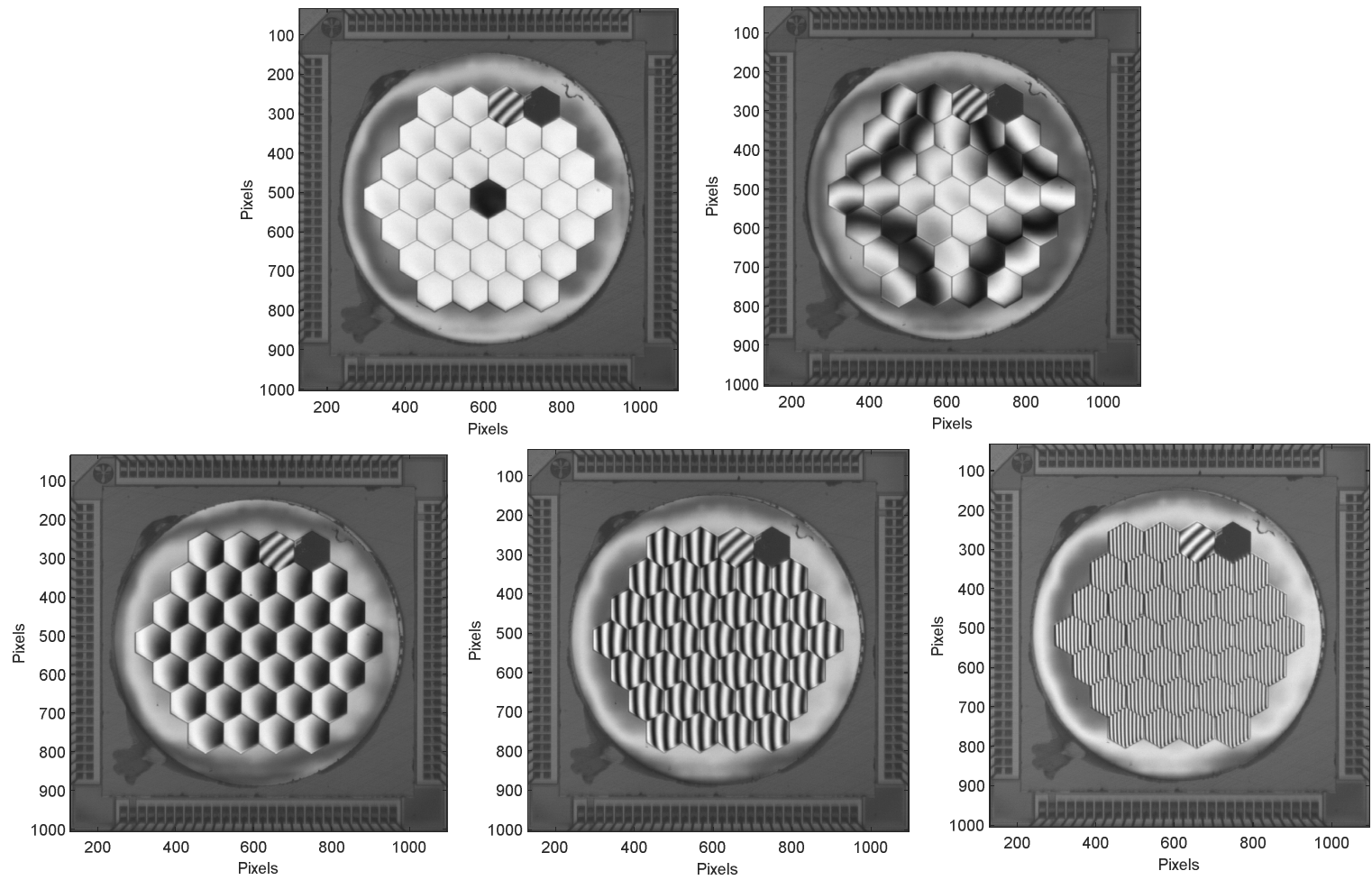

Fig. 6: top left: $150 \mathrm{~nm}$ piston on segment\#1; top right: astigmatism (0.125 on Zernike coefficient);

Bottom: Increasing tilt values along the $\mathrm{X}$ direction are applied to all segments $(0.25,1.5$ and $4.9 \mathrm{mrad})$.

\subsection{Best flat at ambient}

From the interferometric measurement, we obtain the surface deformation shown in Fig. 7. The best flat residual over the whole mirror is very good with $17 \mathrm{~nm}$ RMS, $123 \mathrm{~nm}$ PtV. Note that this best flat provided by Iris AO is not corrected from the gravity effect occurring in our experiment, as the PTT111 is mounted in vertical position.

This result shows the high quality of the mirror architecture and of the fabrication process. This flatness is a combination of a very good reproducibility of the actuator platform position after his elevation thanks to the bimorph flexures (Figure 3), and the choice of thick single-crystalline Silicon for the segment material. This position is very stable; long term measurement has been done at ambient on position stability and reproducibility, but this has not been done yet in cryo.

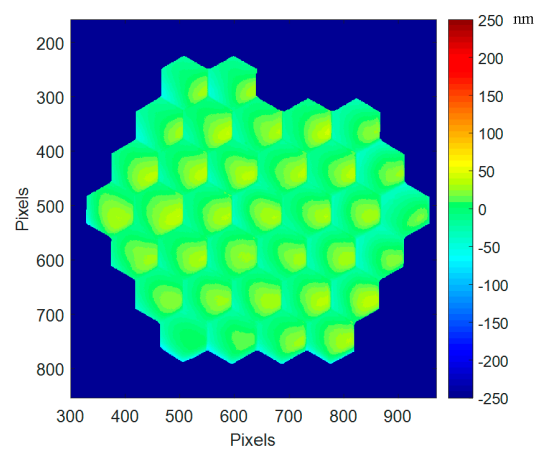

Fig. 7: Best flat surface deformation at ambient (17 nm RMS, $123 \mathrm{~nm} \mathrm{PtV)}$ when the best flat condition calibrated by Iris AO is applied; the gravity effect has not been removed. 
At segment level, the residual wavefront error is with a slight convex shape, observed on most of the segments.

We measure the mirror surface shape with high accuracy and sort out tilt and piston values for each segment: the tilts have a value of $32 \mu \mathrm{rad}$ RMS $(140 \mu \mathrm{rad} \mathrm{PtV})$ while the pistons expands on $6.2 \mathrm{~nm}$ RMS $(28 \mathrm{~nm}$ PtV). The residual tilts are mainly due to the gravity effect as the device is mounted in vertical position in our measurement set-up.

\subsection{Best flat at cryo}

The device is then cooled down slowly from ambient temperature $(293 \mathrm{~K})$ down to $160 \mathrm{~K}$, with the device constantly operating in its best flat condition. The PTT111 device is operating properly at all temperatures between $293 \mathrm{~K}$ and $160 \mathrm{~K}$, and in vacuum.

Every $10 \mathrm{~K}$ an interferometric measurement is done in order to follow the differential deformation of the mirror at whole mirror level as well as at segment level. Several patterns are applied and measured in order to see the ability of the device to behave as at room temperature; the applied "patterns" are best flat, pure pistons on some segments, and different tilts on the segments. Due to the vibrations induced by the cryo pump on the sample, we have to stop it during the measurement, leading to a limited increase of the temperature during the measurement duration. Phase shifting interferometry parameters have been adjusted in order to minimize the measurement time to a few minutes.

In Figure 8, the best flat surface deformation at cryo $(160 \mathrm{~K})$ is given with the original best flat condition as calibrated at ambient. A global convex deformation is observed reaching a deformation of $86 \mathrm{~nm}$ RMS, $501 \mathrm{~nm}$ PtV. Some additional deformations (mainly tilts) are observed on some segments at the upper left side (segments \#26, 27, and 28). The global convex shape in cryo is due to the packaging "shrinking" in cryo. The Coefficient of Thermal Expansion (CTE) mismatch between die/package materials induces a global effect on the mirror when cooled down at $160 \mathrm{~K}$. The mirror is operating perfectly in cryo, and a "new" best flat condition will be developed and described in the next paragraphs.

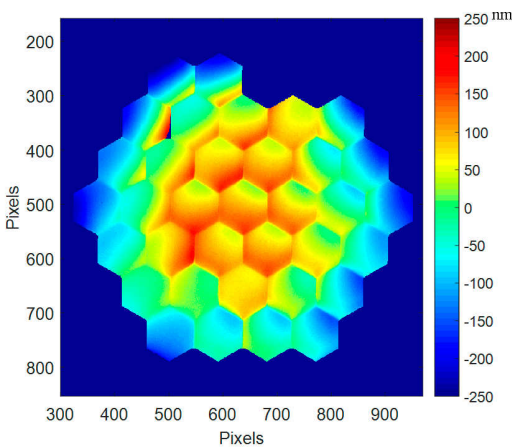

Fig. 8: Best flat mirror deformation at cryo $(160 \mathrm{~K})$, first run, with the original best flat calibrated at ambient (86 nm RMS, $501 \mathrm{~nm}$ PtV).

We measure and sort out for each segment tilt and piston values: the tilts have a value of $200 \mu \mathrm{rad}$ RMS (950 $\mu \mathrm{rad}$ PtV) while the pistons expands on $74 \mathrm{~nm}$ RMS (239 nm PtV). Tilts and pistons are not behaving the same way. Pistons are behaving within the three concentric rings of PTT111 37 segments, with a common motion for the central area (segments \#1 to 7), the middle ring (segments \#8 to 19), and the outer ring (segments \#20 to 37); residual pistons at are $6.2 \mathrm{~nm}$ RMS (28 nm PtV), and $74 \mathrm{~nm}$ RMS (239 nm PTV) in cryo; this effect is clearly related to the shrinkage of the overall device. As for the tilts, they don't show a clear pattern; they are scattered away from the original positions at ambient: residual tilts at ambient are $32 \mu \mathrm{rad}$ RMS (140 $\mu \mathrm{rad}$ PtV), and $200 \mu \mathrm{rad}$ RMS (950 $\mu \mathrm{rad}$ PTV) in cryo; this differential evolution is possibly due to the different modification of the complex structure underneath each segment: the actuator platform, the bonding pads, the mirror segment, the coating, and the underlying 3 legs bimorph structure. ${ }^{11}$

\subsection{Improved best flat at ambient}

When the device is warmed up back to ambient temperature we measure its surface shape in the original best flat mode again, and we get a slightly different result. The degradation of the surface deformation is going up from $17 \mathrm{~nm}$ to $22 \mathrm{~nm}$ RMS (123 nm to $152 \mathrm{~nm} \mathrm{PtV),} \mathrm{the} \mathrm{differences} \mathrm{being} \mathrm{mainly} \mathrm{due} \mathrm{to} \mathrm{the} \mathrm{additional} \mathrm{tilts} \mathrm{on} \mathrm{each} \mathrm{segment} \mathrm{by} 0.15 \mathrm{mrad} \mathrm{PtV}$ over the device, while the pistons stay within the same range as before the cooling cycle. This is probably due to the 
stress relaxation process in the complex structure supporting each segment, leading to a slightly different evolution from segment to segment. As for the segment mirror surface, there is no difference (below $1 \mathrm{~nm}$ ), thanks to the high quality of the segment made of single-crystalline silicon material. This effect could be described as an annealing process. In order to improve the best flat quality, we decided to develop an improved best flat procedure by measuring, with high accuracy, the tip-tilt and piston residuals and combining them with the original best flat values calibrated by Iris AO. We then obtain the improved best flat shown in Figure 9.

The mirror surface deformation is then as low as $10 \mathrm{~nm}$ RMS, $79 \mathrm{~nm} \mathrm{PtV}$.

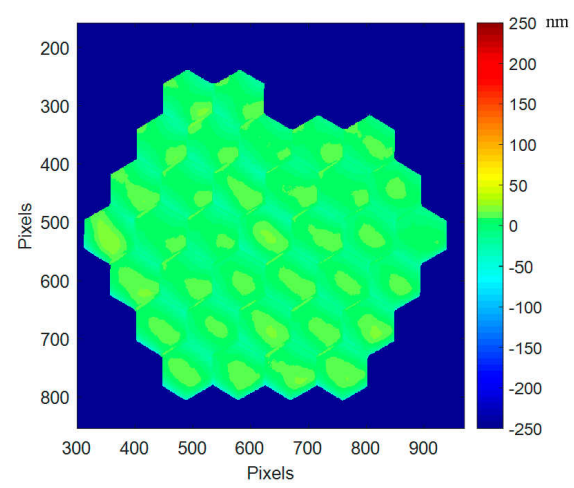

Fig. 9: Mirror surface deformation at ambient (293 K) when improved best flat condition is applied, after one cycle of cooling (10 nm RMS, $79 \mathrm{~nm} \mathrm{PtV}$ )

Residual tilts with improved best flat are $6.5 \mu \mathrm{rad}$ RMS (36 $\mu \mathrm{rad}$ PtV), while they were $32 \mu \mathrm{rad}$ RMS (140 $\mu \mathrm{rad}$ PTV) with the original best flat. Residual pistons with improved best flat are $1.6 \mathrm{~nm}$ RMS (7 nm PtV), while they were $6.2 \mathrm{~nm}$ RMS (28 nm PtV) with the original best flat. ${ }^{11}$

Improved best flat condition will be used in the following experiments.

\subsection{Cryo best flat}

In order to demonstrate full operation of PTT111 at cryogenic temperature, we decide to cool down the device and optimize in-situ all actuators for generating a cryo best flat. Our strategy is a weighted addition of the consecutive measurement residual errors and, using Iris AO electronics, we are loading these calculated values actuator by actuator, departing from the original values provided by Iris AO, and applyling them to the device. Our cryo best flat condition is a combination of [best flat calibrated by Iris-AO at ambient, improved best flat at ambient, improved best flat in cryo (first run), improved best flat in cryo (second run)]. Then, in a single measurement step and applying this best flat condition, we got, at $160 \mathrm{~K}$, a mirror surface deformation as low as $12 \mathrm{~nm} \mathrm{RMS,} 113 \mathrm{~nm}$ PtV (Figure 10).

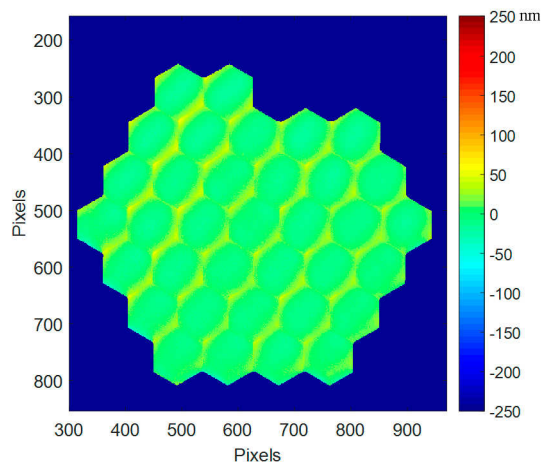

Fig. 10: Mirror surface deformation at cryo $(160 \mathrm{~K})$, second run, when cryo best flat condition is applied (12 nm RMS, 113 nm PtV); 
Our cryo best flat at $160 \mathrm{~K}$ is then very close to our improved best flat at ambient (293 K), showing our ability to operate properly PTT111 device in cryo. The deformation difference is $2 \mathrm{~nm} \mathrm{RMS,} 34 \mathrm{~nm} \mathrm{PtV}$ between $160 \mathrm{~K}$ and $293 \mathrm{~K}$. This additional deformation is due mainly to the mirror segment deformation, as revealed in the following Section 4.5.

Residual tilts with cryo best flat are $3.5 \mu \mathrm{rad}$ RMS (17 $\mu \mathrm{rad}$ PtV), while they were $6.5 \mu \mathrm{rad}$ RMS (36 $\mu \mathrm{rad}$ PtV) with the improved best flat at ambient. Residual pistons with cryo best flat are $1.2 \mathrm{~nm}$ rms $(4.3 \mathrm{~nm} \mathrm{PtV})$, while they were $1.6 \mathrm{~nm}$ RMS ( $7 \mathrm{~nm} \mathrm{PtV)}$ with the improved best flat at ambient. ${ }^{11}$

A second loop of best flat optimisation is useless as the remaining mirror surface deformation is only due to the contributions of individual segment deformations; this is clearly visible in the surface deformation map of Figure 10. The additional deformation of PTT111 at cryo is $501 \mathrm{~nm}$ PtV (Figure 8). The cryo best flat is compensating this deformation, minimizing the whole mirror deformation, down to $12 \mathrm{~nm}$ RMS (123 nm PtV). The maximum stroke of this device being $3.01 \mu \mathrm{m}$ at ambient, then the operational stroke is reduced to $2.5 \mu \mathrm{m}$ at cryo (16.7\% stroke reduction).

\subsection{Segment characterization}

Thanks to our set-up spatial resolution, we have several thousand measurement points per segment. It is then possible to measure, at segment level, the deformation induced by the strong temperature change from ambient to cryo. The surface deformation by segments at ambient has a mean value of $7.2 \mathrm{~nm}$ with a standard deviation of $1.5 \mathrm{~nm}$, and at $160 \mathrm{~K}$, a mean value of $8.5 \mathrm{~nm}$ with a standard deviation of $1.6 \mathrm{~nm}$.

By selecting a typical segment, a closer analysis of the segment evolution at cryogenic temperature could be done, especially on its shape. In Figure 11, on segment \#21, we can clearly see that the convex cylindrical shape at ambient is changing to an astigmatic concave shape at cryo. At ambient (293 K), the segment surface deformation is $5 \mathrm{~nm}$ RMS (24 nm PtV), while at $160 \mathrm{~K}$ the deformation is still low, at $8 \mathrm{~nm} \mathrm{RMS} \mathrm{(47} \mathrm{nm} \mathrm{PtV).}$

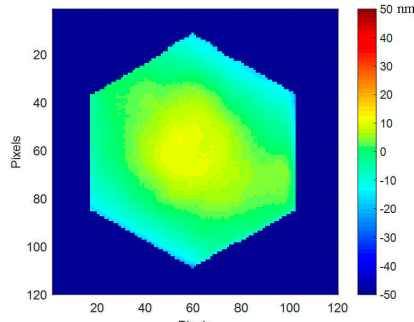

(a)

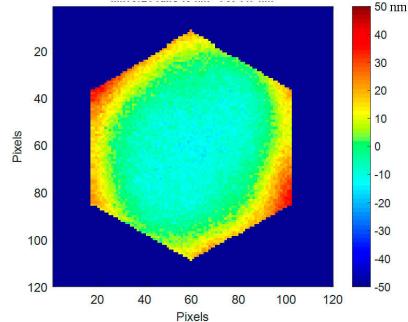

(b)

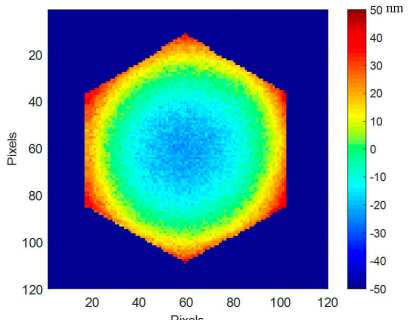

(c)

Fig. 11: DM segment \#21 surface deformation: (a) At ambient (293 K), $5 \mathrm{~nm} \mathrm{RMS,} 24 \mathrm{~nm} \mathrm{PtV;}$ (b) At $160 \mathrm{~K} 8 \mathrm{~nm}$ RMS, $47 \mathrm{~nm} \mathrm{PtV}$; (c) Deformation difference between ambient and $160 \mathrm{~K}, 4.9 \mathrm{~nm} \mathrm{RMS,} 71 \mathrm{~nm}$ PtV.

A very interesting feature is observed when looking at the deformation difference between ambient and $160 \mathrm{~K}$ (Figure 11c): it reveals a pure concave axisymetrical change of $4.9 \mathrm{~nm} \mathrm{RMS} \mathrm{(71} \mathrm{nm} \mathrm{PtV).} \mathrm{This} \mathrm{is} \mathrm{due} \mathrm{to} \mathrm{the} \mathrm{CTE}$ mismatch between the single-crystalline silicon and the silver-protected coating deposited on top of the segment. All segments are behaving in the same way; the mean deformation at ambient is in the range of $25 \mathrm{~nm}$, while it rises to $50 \mathrm{~nm}$ in cryo. This deformation difference is still within the requirement of almost all foreseen wavefront correction systems. This deformation difference at segment level is the major contribution to the whole mirror surface deformation, as described in Section 4.4. ${ }^{11}$ 


\section{CONCLUSION}

Innovative wavefront correction systems in existing telescopes on the ground and in space, as well as in the future ELTs, need efficient MOEMS Deformable Mirrors (DM) able to perform at room temperature as well as in cryogenic and vacuum environments.

Using a specific interferometric bench coupled with a cryo-vacuum chamber, a PTT 111 DM from Iris AO has been successfully tested from ambient temperature to $160 \mathrm{~K}$. The device is properly operating in cryo, revealing an additional, mainly focus-like, $500 \mathrm{~nm}$ deformation at $160 \mathrm{~K}$; we were able to recover the best flat in cryo by correcting the focus and local tip-tilts on all segments, reaching $12 \mathrm{~nm}$ RMS on the entire surface.

Finally, the goal of these studies is to test DMs in cryo and vacuum conditions as well as to improve their architecture for stable operation in a harsh environment.

\section{REFERENCES}

[1] F. Zamkotsian, K. Dohlen, "Prospects for MOEMS-based adaptive optical systems on extremely large telescopes", in Proceedings of the conference Beyond conventional Adaptive Optics, Venice, Italy (2001)

[2] S. Waldis, F. Zamkotsian, P.-A. Clerc, W. Noell, M. Zickar, N. De Rooij, "Arrays of high tilt-angle micromirrors for multiobject spectroscopy, " IEEE Journal of Selected Topics in Quantum Electronics 13, pp. 168-176 (2007).

[3] M. Canonica, F. Zamkotsian, P. Lanzoni, W. Noell, N. de Rooij, "The two-dimensional array of 2048 tilting micromirrors for astronomical spectroscopy," Journal of Micromechanics and Microengineering, 23055009 , (2013)

[4] Frederic Zamkotsian, Harald Ramarijaona, Manuele Moschetti, Patrick Lanzoni, Marco Riva, Nicolas Tchoubaklian, Marc Jaquet, Paolo Spano, William Bon, Romain Alata, Luciano Nicastro, Emilio Molinari, Rosario Cosentino, Adriano Ghedina, Manuel Gonzalez, Walter Boschin, Paolo Di Marcantonio, Igor Coretti, Roberto Cirami, Filippo Zerbi, Luca Valenziano, "Building BATMAN: a new generation spectro-imager on TNG telescope ", in Proceedings of the SPIE conference on Astronomical Instrumentation 2016, Proc. SPIE 9908, Edinburgh, United Kingdom, (2016)

[5] G. Vdovin, S. Middelhoek and P. M. Sarro, "Technology and applications of micromachined silicon adaptive mirrors", Opt. Eng., 36 (5), 1382-1390 (1997)

[6] M.A. Helmbrecht, M. He, C.J. Kempf, F. Marchis "Long-term stability and temperature variability of Iris AO segmented MEMS deformable mirrors”, Proc. SPIE 9909, (2016)

[7] S. Cornelissen, T. G. Bifano "Advances in MEMS deformable mirror development for astronomical adaptive optics", in Proceedings of the SPIE conference on MOEMS 2012, Proc. SPIE 8253, San Francisco, USA (2012)

[8] A. Liotard, F. Zamkotsian, "Static and dynamic micro-deformable mirror characterization by phase-shifting and time-averaged interferometry", in Proceedings of the SPIE conference on Astronomical Telescopes and Instrumentation 2004, Proc. SPIE 5494, Glasgow, United Kingdom (2004)

[9] F. Zamkotsian, E. Grassi, S. Waldis, R. Barette, P. Lanzoni, C. Fabron, W. Noell, N. de Rooij, " Interferometric characterization of MOEMS devices in cryogenic environment for astronomical instrumentation," Proc. SPIE 6884, San Jose, USA, (2008)

[10] F. Zamkotsian, , P. Lanzoni, R. Barette, E. Grassi, P. Vors, M. Helmbrecht, F. Marchis, A. Teichman, "MOEMS deformable mirror testing in cryo for future optical," Proc. SPIE 10116, San Francisco, USA, (2017)

[11] F. Zamkotsian, , P. Lanzoni, R. Barette, M. Helmbrecht, F. Marchis, A. Teichman, " Operation of a MOEMS Deformable Mirror in Cryo: Challenges and Results," Micromachines 8, 233; doi:10.3390/mi8080233 (2017) 